\title{
On-Body Calibration and Measurements Using Personal Radiofrequency Exposimeters in Indoor Diffuse and Specular Environments
}

\author{
Reza Aminzadeh, ${ }^{1 *}$ ArnoThielens, ${ }^{1}$ Aliou Bamba, ${ }^{1,2}$ Lamine Kone, ${ }^{2}$ \\ Davy Paul Gaillot, ${ }^{2}$ Martine Lienard, ${ }^{2}$ Luc Martens, ${ }^{1}$ and Wout Joseph ${ }^{1}$ \\ ${ }^{1}$ Department of Information Technology, Ghent University/iMinds, Ghent, Belgium \\ ${ }^{2}$ Institut d'Electronique, de Microelectronique et de Nanotechnologie (IEMN), University \\ of Lille, Lille, France
}

\begin{abstract}
For the first time, response of personal exposimeters (PEMs) is studied under diffuse field exposure in indoor environments. To this aim, both numerical simulations, using finite-difference timedomain method, and calibration measurements were performed in the range of $880-5875 \mathrm{MHz}$ covering 10 frequency bands in Belgium. Two PEMs were mounted on the body of a human male subject and calibrated on-body in an anechoic chamber (non-diffuse) and a reverberation chamber (RC) (diffuse fields). This was motivated by the fact that electromagnetic waves in indoor environments have both specular and diffuse components. Both calibrations show that PEMs underestimate actual incident electromagnetic fields. This can be compensated by using an on-body response. Moreover, it is shown that these responses are different in anechoic chamber and RC. Therefore, it is advised to use an on-body calibration in an RC in future indoor PEM measurements where diffuse fields are present. Using the response averaged over two PEMs reduced measurement uncertainty compared to single PEMs. Following the calibration, measurements in a realistic indoor environment were done for wireless fidelity (WiFi-5G) band. Measured power density values are maximally $8.9 \mathrm{~mW} / \mathrm{m}^{2}$ and $165.8 \mu \mathrm{W} / \mathrm{m}^{2}$ on average. These satisfy reference levels issued by the International Commission on Non-Ionizing Radiation Protection in 1998. Power density values obtained by applying on-body calibration in RC are higher than values obtained from no body calibration (only PEMs) and on-body calibration in anechoic room, by factors of 7.55 and 2.21 , respectively. Bioelectromagnetics. 2016;9999:XX-XX. C C 2016 Wiley Periodicals, Inc.
\end{abstract}

Key words: personal exposimeters; human exposure; electromagnetic fields; diffuse fields; indoor environment

\section{INTRODUCTION}

The increasing number of radiofrequency (RF) electromagnetic sources in the environment has resulted in a continuous and growing concern about health effects caused by these fields to which humans are exposed. In recent years, a large number of studies have investigated continuous everyday life RF exposures [Röösli et al., 2008; Joseph et al., 2008, 2010; Bolte and Eikelboom, 2012; Thielens et al., 2013]. To this end, portable personal exposimeters (PEM) were designed to continuously monitor exposure of a human subject. For such measurements, measurement protocols have been developed [Joseph et al., 2008; Mann, 2010; Röösli et al., 2010]. In Bolte et al. [2011], Iskra et al. [2010], and Thielens et al. [2015a], it was shown that PEMs are faced with measurement uncertainties caused by presence of user's body. As discussed in Thielens et al. [2015a], PEMs are calibrated in free space (no body calibration in absence of a person), while used on body. Therefore, measured electric field values might deviate from actual incident field values for which reference levels issued by the International Commission on Non-Ionizing Radiation Protection (ICNIRP) [1998]. Therefore, correct calibration of PEMs is necessary.

Grant sponsor: Research Foundation-Flanders (FWO-V); grant number: G003415N.

*Correspondence to: Reza Aminzadeh, Ghent UniversityINTEC, Gaston Crommenlaan 8, B-9050 Ghent, Belgium. E-mail: reza.aminzadeh@intec.ugent.be

Received for review 25 September 2015; Accepted 11 March 2016

DOI: 10.1002/bem.21975

Published online XX Month Year in Wiley Online Library (wileyonlinelibrary.com). 
Indoor environments are realistic environments where people spend most of their time and are exposed to several RF electromagnetic sources simultaneously. According to the room electromagnetics theory [Andersen et al., 2007, 2012], total power in an indoor environment is composed of non-diffuse (specular; i.e., line-of-sight (LOS) component) and diffuse multipath components (DMC). The former is a result of coherent reflections, while the latter is a result of non-coherent reflections from objects in the room. In other words, specular components are the result of reflection from large surfaces (e.g., walls) in a room, while due to irregularities at surfaces and presence of other objects, refraction occurs and consequently reflected waves consist of specular components and a set of scattered waves, which have different properties than reflected specular component. DMC cannot be characterized with specular paths. Multiple sets of such diffracted waves result in a diffuse field in an indoor environment. Previous studies have shown that contribution of DMC to total power [Poutanen et al., 2011] and to total whole-body averaged specific absorption rate [Bamba et al., 2013] may increase up to $95 \%$ and $88 \%$ in an indoor environment, respectively. Therefore, it is necessary to assess exposure of humans to various RF sources under diffuse field conditions.

Large variations in response of PEMs have been reported due to difference in angle of arrival for incident waves (35 dB) [Bahillo et al., 2008] or caused by the human body ( $95 \%$ confidence interval of $18 \mathrm{~dB}$ at $900 \mathrm{MHz}$ ) [Iskra et al., 2011]. In contrast, it was shown that using more than one PEM on the body can reduce measurement uncertainties significantly [Thielens et al., 2013]. In Bolte et al. [2011], the effect of the body on measurements was reduced by calibration of PEMs in an open area.

As discussed above, the number of studies on PEMs' response in realistic environments has increased in recent years, but none of these studies focused on human exposure to diffuse fields, which are dominant in indoor environments. To the best of authors' knowledge, numerical simulations and experimental measurements for assessment of the response of PEMs in diffuse fields have not been published yet.

The goal of this article is to determine, for the first time, whether PEMs can be used for reliable onbody measurements in diffuse fields. To this aim, the response of PEMs in diffuse fields (in indoor environments) was studied for the first time both numerically and experimentally in the range of $880-5875 \mathrm{MHz}$ covering 10 frequency bands in Belgium. We calibrated two PEMs worn on the body at the same time in an anechoic chamber and in a reverberation chamber (RC) under exposure to non-diffuse and diffuse fields, respectively. Anechoic chamber provides damping of reflected waves for studied frequency bands, while the RC is a closed metallic cavity in which diffuse fields have statistically uniform distributions for all measurement points [Andersen et al., 2007]. The two-PEM calibration in both rooms (specular and diffuse) was proposed to determine the PEM's response, which can be used to estimate actual incident electromagnetic field strength from on-body measurements using PEMs. We will show that these responses were different in diffuse and specular fields. Calibrated PEMs were then used to perform real measurements for an indoor application of wireless fidelity around $5 \mathrm{GHz}$ (WiFi-5G), since this frequency band has not often been investigated yet and to date no on-body calibration of PEMs has been done for this band. These measurements are faced with uncertainties, which can also be determined using proposed calibration procedure. We will show that using two PEMs simultaneously reduced measurement uncertainty of measured incident field strength in realistic environments.

\section{MATERIALS AND METHODS}

Figure 1 shows a flow graph of proposed methodology. The goal of this study was to execute measurements of the RF personal exposure in an indoor environment using PEMs. To this aim, an onbody calibration was executed in both an anechoic chamber and a reverberation chamber. Then calibrations resulted in responses $(R)$ in either specular or diffuse fields. These responses can be used to estimate incident E-field strength from the PEM measurements.

\section{Personal Exposimeters (PEMs)}

In this study, two EME SPY140 (Satimo, Brest, France) PEMs were used. These PEMs can measure a wide range of frequency bands from frequency modulation (FM) to WiFi-5G. Detection limit of these PEMs is $0.005 \mathrm{~V} / \mathrm{m}$ for frequencies below $3 \mathrm{GHz}$ and $0.02 \mathrm{~V} / \mathrm{m}$ for frequencies higher than $3 \mathrm{GHz}$ with a maximum sample rate of $0.25 \mathrm{~Hz}$. In this study, we focused on those bands above $800 \mathrm{MHz}$. Studied frequency bands are listed in Table 1.

\section{Numerical Simulations}

The goal of simulations was to determine distribution of received power on a pair of dipoles worn on the body in diffuse fields. Also location of PEMs on the body is a parameter that affects their response. To study response of PEMs on the body, 


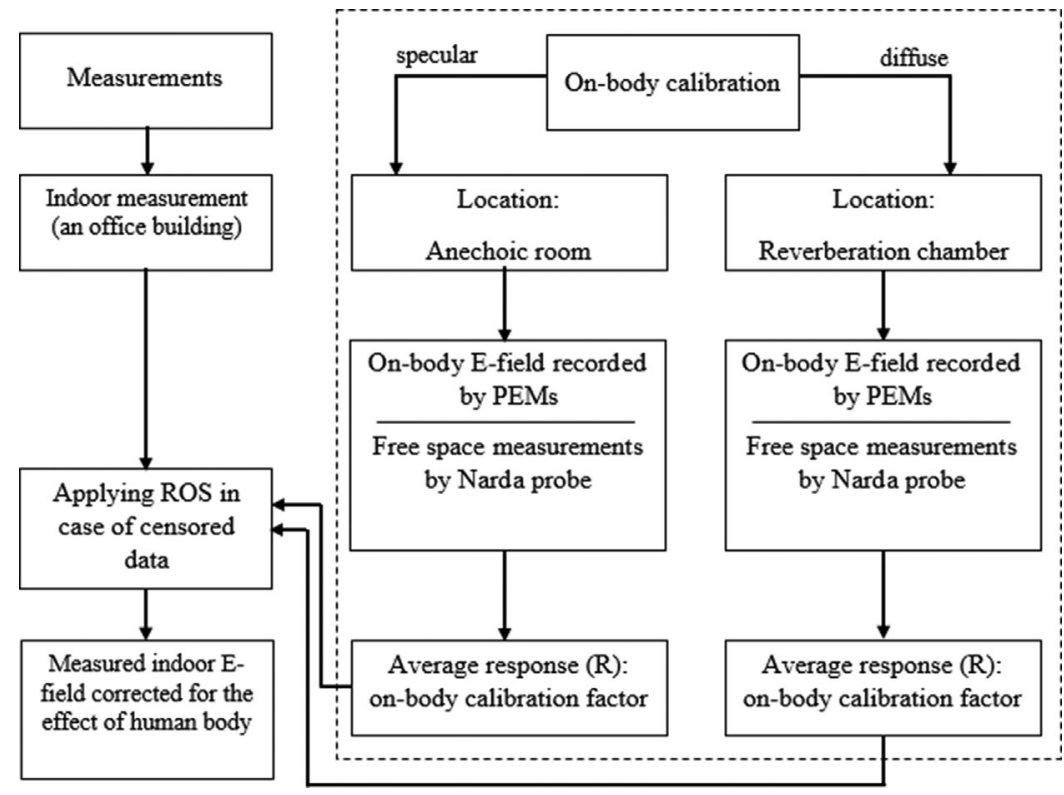

Fig. 1. Flow graph of proposed methodology.

finite-difference time-domain (FDTD) simulations were performed to model the reverberation chamber environment. For this purpose, the SEMCAD-X simulation platform [SPEAG, 2009] was used. To model each PEM, a dipole antenna was placed next to a heterogeneous human body model called virtual family male (VFM) [Christ et al., 2010] consisting of 81 different tissues and has a body mass index (BMI) of $22.3 \mathrm{~kg} / \mathrm{m}^{2}$. A grid step of $1.5 \mathrm{~mm}$ was used to appropriately resolve the skin of the numerical model. Dielectric properties used for this phantom were taken from Gabriel et al. [1996]. Chosen locations for mounting PEMs (dipoles) were the front and back of the body, since in reality, at these locations perfor- mance of PEMs would be less affected by user movements. Moreover, using a PEM at each side of body trunk minimizes measurement uncertainty [Thielens et al., 2015a]. Dipole antennas were placed at $19 \mathrm{~mm}$ from the front and the back of the body, as this is half the width of the EME SPY140 commercial exposimeters.

Numerical simulations were performed in the range of $880-5875 \mathrm{MHz}$ covering 10 frequency bands from GSM900-UL to WiFi-5G in Belgium [BIPT, 2015]. For each frequency band, center frequency was selected for numerical simulations and a dipole antenna was designed. Length of each antenna was optimized at a separation of $19 \mathrm{~mm}$ from a lossy homogenous

TABLE 1. The Studied Frequency Bands and Their Measured Center Frequencies, the Optimized Length of Dipoles, and the Design Coefficients for Each RF Signal

\begin{tabular}{lcccc}
\hline RF signal & $\begin{array}{c}\text { Frequency range } \\
(\mathrm{MHz})\end{array}$ & $\begin{array}{c}\text { Center frequency } \\
(\mathrm{MHz})\end{array}$ & $\begin{array}{c}\text { Optimized length of dipoles } L \\
(\mathrm{~mm})\end{array}$ & $\begin{array}{c}\text { Design coefficient } \\
(\mathrm{\iota})\end{array}$ \\
\hline GSM900-UL & $880-915$ & 897 & 151 & 0.45 \\
GSM900-DL & $925-960$ & 950 & 142.6 & 0.45 \\
GSM1800-UL & $1710-1785$ & 1748 & 73.7 & 0.43 \\
GSM1800-DL & $1805-1880$ & 1843 & 69.9 & 0.43 \\
DECT & $1880-1900$ & 1890 & 67.4 & 0.42 \\
UMTS-UL & $1920-1980$ & 1950 & 65.3 & 0.42 \\
UMTS-DL & $2110-2170$ & 2140 & 59.1 & 0.42 \\
WiFi-2G & $2400-2483.5$ & 2450 & 50.5 & 0.41 \\
WIMAX & $3400-3600$ & 3500 & 33.8 & 0.39 \\
WiFi-5G & $5150-5875$ & 5500 & 21.2 & 0.39 \\
\hline
\end{tabular}

GSM, global system for mobile communications; DECT, digital enhanced cordless telecommunications; UMTS, universal mobile telecommunications system; WiFi, wireless fidelity; WIMAX, worldwide interoperability for microwave access, UL, uplink; DL, downlink. 
phantom $\left(40 \times 20 \times 15 \mathrm{~cm}^{3}\right)$, with dielectric properties of muscle taken from Gabriel et al. [1996]. This was done to ensure that in presence of the VFM phantom, antennas have a return loss $\left|S_{11}\right|^{2}$ lower than $-10 \mathrm{~dB}$ at each center frequency. A design coefficient $(\iota)$ is extracted for the antennas as $(L=\iota \times \lambda)$, where $(L)$ is optimized total length of antenna and $(\lambda)$ is the wavelength. Table 1 lists optimized length of the dipole antennas versus design coefficient as a function of frequency for the studied frequency bands. It can be seen that ( $\iota$ ) decreases as frequency increases and is in the range of 0.39-0.45. This is in good agreement with $0.47 \lambda$, which is the length of the dipoles used near a body phantom at $2.4 \mathrm{GHz}$ [Roelens et al., 2008].

In order to simulate exposure to diffuse fields, FDTD was used to perform simulations of the VFM equipped with two dipoles under exposure of single plane waves. Then the approach of Thielens et al. [2015b] was applied to combine different single plane waves using sets of multiple plane waves to calculate received power on each dipole and emulate realistic scenarios. According to Andersen et al. [2007], angles of incidence of the diffuse fields that are incident on an antenna have a uniform distribution. Therefore, a set of 2000 plane waves were generated with incident angles drawn from uniform distributions for both the azimuth angle $(\varphi): 0<\varphi<2 \pi$, and polar angle $(\theta)$ : $0<\theta<\pi$. Polarization angle $(\psi)$ of plane waves was drawn from a uniform distribution between $0<\psi<\pi$ and the phase $(\alpha)$ of plane waves was drawn from a uniform distribution between $0<\alpha<2 \pi$. Fields were assumed to have a constant amplitude equal to $1 \mathrm{~V} / \mathrm{m}$ [Andersen et al., 2007; Bamba et al., 2012].

Received power on an antenna $\left(P_{r}\right)$ (in diffuse fields) can be determined from its aperture [Balanis, 2005]:

$$
P_{r}(\varphi, \theta)=A A(\varphi, \theta) \times S_{\mathrm{inc}}
$$

where $A A$ and $\mathrm{S}_{\text {inc }}$ are on-body antenna aperture and incident power density, respectively. In order to calculate the on-body aperture of dipoles $A A(\varphi, \theta)$, directive gain $D(\varphi, \theta)$ of each antenna is extracted for azimuth angle $(\varphi)$ and polar angle $(\theta)$ from (single plane wave) numerical simulations as:

$$
A A(\varphi, \theta)=\eta_{\mathrm{rad}}\left(1-\left|S_{11}\right|^{2}\right) D(\varphi, \theta) \frac{\lambda^{2}}{4 \pi}
$$

where $\eta_{\text {rad }}$ is radiation efficiency, $\left|S_{11}\right|^{2}$ is antenna's power reflection coefficient, and $\lambda$ is wavelength. $A A$ $(\varphi, \theta)$ can be determined for two orthogonal polarizations on the incident electric fields: $\theta$ and $\varphi$, which are the polarizations parallel to the unity vectors $\overline{1}_{\theta}$ and $\overline{1}_{\varphi}$. For multiple plane waves incident on the antenna, received power on the antenna is not necessarily equal to the sum of incident powers induced by each single plane wave, since the incident plane waves can interfere with each other. Therefore, received power should be calculated as a function of incident electric fields (sum of induced voltages on dipoles):

$$
\begin{aligned}
V_{r}=\sum_{j=1}^{N_{p w}}\left[A F\left(\theta_{j}, \varphi_{j}, 0^{\circ}\right)\right. & \times\left(\bar{E}_{i n c, j}\left(\theta_{j}, \varphi_{j}, \psi_{j}\right) \cdot \overline{1}_{\theta}\right)+A F\left(\theta_{j}, \varphi_{j}, 90^{\circ}\right) \\
& \left.\times\left(\bar{E}_{i n c, j}\left(\theta_{j}, \varphi_{j}, \psi_{j}\right) \cdot \overline{1}_{\varphi}\right)\right] \times e^{\alpha j}
\end{aligned}
$$

where $V_{r}$ is received voltage on the dipole, $\bar{E}_{i n c, j}\left(\theta_{j}, \varphi_{j}, \psi_{j}\right)$ is incident electric field of plane wave $\mathrm{j}$ with polar angle $\theta$, azimuth angle $\varphi$, polarization $\psi_{j}$, amplitude $\left|\bar{E}_{i n c, j}\right|$, phase $\alpha$, and $N_{p w}$ is number of incident plane waves. Antenna factor $A F\left(\theta_{j}, \varphi_{j}, \psi_{j}\right)$ defined as:

$$
A F\left(\theta_{j}, \varphi_{j}, \psi_{j}\right)=\sqrt{A A\left(\theta_{j}, \varphi_{j}, \psi_{j}\right) \times \frac{|Z|}{377}}
$$

where $A A\left(\theta_{j}, \varphi_{j}, \psi_{j}\right)$ is the antenna aperture and is obtained from Equation (2). Received power $\left(P_{r}\right)$ is obtained as:

$$
P_{r}=\frac{V_{r} \times V_{r}^{*}}{|Z|}
$$

where $V_{r}^{*}$ is complex conjugate of received voltage and $|\mathrm{Z}|$ is magnitude of input impedance of antenna.

\section{Calibration Measurement Setup}

The goal of on-body calibrations was to compare actual fields with and without the body and to test whether PEMs could record reliable data in indoor environments. Calibration measurements consist of two types of measurements; free-space and on-body measurements. Since total power in an indoor environment consists of both specular components (result of specular reflections) and diffuse fields, on-body calibration measurements were performed in an anechoic chamber (specular or non-diffuse) and in a reverberation chamber (diffuse), separately.

\section{Measurement Setup in the Anechoic Chamber}

Figure 2a shows setup for measurements in anechoic chamber. On-body calibration measurements were done in an anechoic chamber representing a specular environment following routines proposed in 

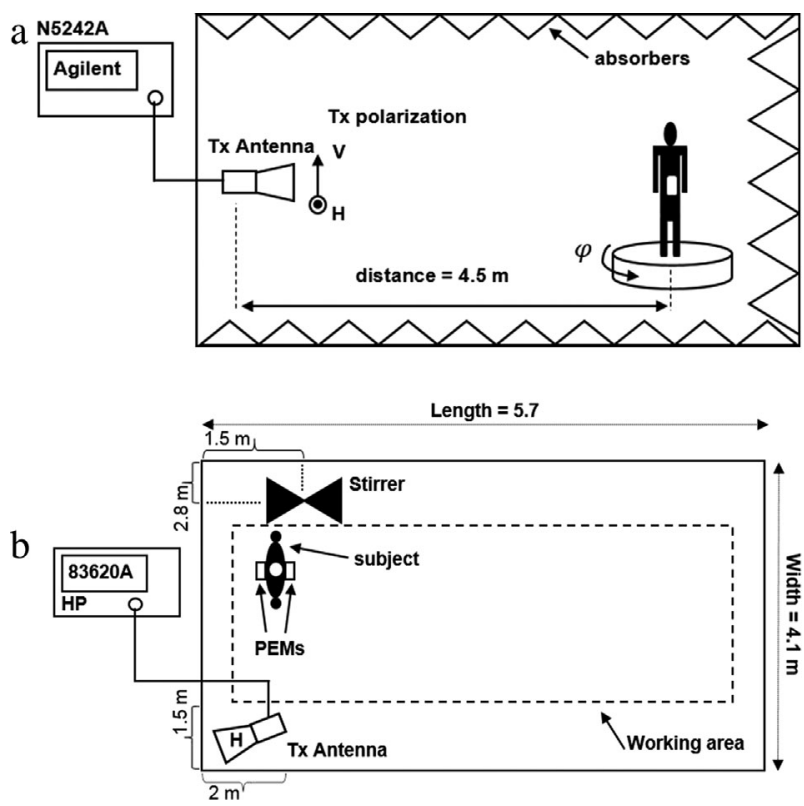

Fig. 2. (a) Measurement setup in anechoic chamber (side view). The white rectangle indicates location of PEMs on front and on back of subject's body. (b) Measurement setup in the reverberation chamber (top view)

Bolte et al. [2011] and Thielens et al. [2015a]. The anechoic chamber used in this study had sufficient damping for frequencies higher than $800 \mathrm{MHz}$. As a result, only frequencies higher than $800 \mathrm{MHz}$ were studied. A vector network analyzer (VNA) (N5242A, Agilent, Santa Clara, CA) was used as a continuous wave $(\mathrm{CW})$ source to deliver a constant power in the range of $31-63 \mathrm{~mW}$ (all results were normalized to $10 \mathrm{~mW}$ ) at the center frequency of each considered band to a transmitting horn antenna (TX) (Scientific Atlanta, Lawrenceville, GA) with a reflection coefficient lower than $-10 \mathrm{~dB}$ in the range of studied frequency bands. Two orthogonal polarizations of the antenna were studied, horizontal $(\mathrm{H})$ and vertical $(\mathrm{V})$, which were parallel to the floor of anechoic chamber and to the axis of rotational platform, respectively.

In the first step, free-space electric field measurements were performed at different heights from the rotational platform in the range of 54-203 cm, using a broadband field meter NBM-550 in combination with EF 0391/EF 0691 probes (all from Narda Safety Test Solutions, Hauppauge, NY). Free space incident electric field $\left(E_{R M S}^{f r e e}\right)$ was then averaged over the total height [ICNIRP, 1998].

In the second step, a 27-year-old male subject with height of $181 \mathrm{~cm}$, mass of $75 \mathrm{~kg}$, and body mass index of $22.9 \mathrm{~kg} / \mathrm{m}^{2}$ stood on the rotational platform in the anechoic chamber and two PEMs were placed on the front and back of his body. The subject was then rotated over $360^{\circ}$ in the azimuthal angle $(\varphi)$ for both $(\mathrm{H}$ and $\mathrm{V})$ polarizations. The same constant power was emitted by the TX at center frequency $f_{\mathrm{j}}$ of each band (see Table 1). Incident electric field was measured by both PEMs for every angle of $(\varphi)$, resulting in a distribution for the recorded electric field by each PEM [Bahillo et al., 2008; Bolte et al., 2011; Iskra et al., 2011]. Each measurement was performed during $240 \mathrm{~s}$ at a sample rate of $0.25 \mathrm{~Hz}$ for every $f_{\mathrm{j}}$ and $\varphi$. This way, the effect of random orientation of the subject in realistic environments was included in the calibration measurements [Thielens et al., 2015a]. Finally, response $(R)$ of PEMs was determined as:

$$
R_{i j}\left(f_{j}, \varphi\right)=\left(\frac{E_{R M S, i j}^{b o d y}\left(f_{j}, \varphi\right)}{E_{R M S}^{f r e e}\left(f_{j}\right)}\right)^{2}
$$

where $E_{R M S, i j}^{b o d y}\left(f_{j}, \varphi\right)$ is recorded on-body electric field by each PEM ( $\mathrm{i}=$ front or back) when a signal is emitted at $f_{\mathrm{j}}$ in the same band by the transmitting antenna. Ideally, a PEM worn on body should record $E_{R M S}^{\text {free }}$ (actual value), hence $R$ should be 1 . However, in reality electric field recorded by a PEM is lower or higher than $E_{R M S}^{\text {free }}$ due to presence of human body. Therefore $R$ can be lower than 1 (underestimation) or higher than 1 (overestimation). The average response $\left(R_{\text {avg, }, j}\right)$ in the frequency band $\mathrm{j}$ is defined as:

$$
R_{\text {avg }, j}\left(f_{j}, \varphi\right)=1 / 2 \times\left(R_{\text {front }, j}\left(f_{j}, \varphi\right)+R_{\text {back }, j}\left(f_{j}, \varphi\right)\right)
$$

where $R_{\text {front/back }}\left(f_{j}, \varphi\right)$ is response measured by PEMs on the front and on back of body, respectively. Here $p_{2.5}, p_{25}, p_{75}$, and $p_{97.5}$ are the $2.5 \%, 25 \%, 75 \%$, and $97.5 \%$ percentiles of responses. The interquartile distance $\left(P I_{50}\right)$ of the response is the ratio of $p_{75}$ to $p_{25}$ percentiles. The $95 \%$ prediction interval $\left(P I_{95}\right)$, is the ratio of $p_{97.5}$ to $p_{2.5}$ percentiles of response.

Another parameter to assess calibration measurements is the crosstalk $\left(C_{i j k}\right)$, which is defined as:

$$
C_{i j k}=\left(\frac{E_{R M S, i k}^{b o d y}\left(f_{j}, \varphi\right)}{E_{R M S}^{f r e e}\left(f_{j}\right)}\right)^{2}
$$

With value of $E_{R M S, i k}^{b o d y}\left(f_{j}, \varphi\right)$, response is recorded in band $k$ when signal is emitted in band $\mathrm{j}$. This quantity shows fraction of power received in a specific frequency band $(k)$ but emitted in another band $(\mathrm{j})$. The ideal crosstalk is $C_{i j k}=\delta_{j k}$, that is $\overline{\bar{C}}_{i}=\overline{\overline{1}}$, with $\overline{\overline{1}}$ the unity matrix. 


\section{Measurement Setup in RC}

Figure $2 b$ shows an illustration of the setup for measurements in the RC. The working area of the chamber where fields have uniform distribution [Bamba et al., 2015] is indicated with dashed lines. Room dimensions are $5.7 \times 4.1 \times 2.8 \mathrm{~m}^{3}$ (length $\times$ width $\times$ height) which provides a volume equal to $65 \mathrm{~m}^{3}$. A stirrer composed of four rotating metallic sheets is placed in the chamber to stir electromagnetic modes. A SAS-571 horn antenna (A.H Systems, Chatsworth, CA) operating in the range of the studied frequency bands was used.

In the first step, a broadband field meter NBM550 in combination with EF 0391/EF 0691 probes (all from Narda Safety Test Solutions, Hauppauge, NY) was used at different heights from the floor $(53-203 \mathrm{~cm})$ and measured free-space incident electric field $\left(E_{R M S}^{f r e e}\right)$ was averaged over total measured height. A HP83620A signal generator (Hewlett-Packard, Palo Alto, CA) was used to deliver a constant power of $10 \mathrm{~mW}$ at each center frequency $f_{\mathrm{j}}$ (see Table 1) to the SAS-571 (TX) with horizontal polarization. The incident electric field was measured while the stirrer rotated from $0^{\circ}$ to $360^{\circ}$ (with a step of $6^{\circ}$ ). To check uniform distribution of diffuse fields inside RC, measurements were repeated for studied frequency bands (j) with a vertically polarized TX for two selected heights. The measurement performed to characterize the uniformity region of RC are not shown in this article.

In the second step, the subject was placed inside the RC at the same location as the NBM-550 probe. Two PEMs were placed on the front and back of the subject's body and a constant power of $1 \mathrm{~mW}$ was delivered to the TX at the same center frequencies $\left(f_{\mathrm{j}}\right)$. PEMs measured electric field strengths on the body, while the stirrer rotated from $0^{\circ}$ to $360^{\circ}$ with a step of $1^{\circ}$. During one rotation, 90 samples were recorded at a sample rate of $0.25 \mathrm{~Hz}$. The average response $\left(R_{a v g, j}\right)$ was obtained for studied frequency bands from equations (6) and (7).

Response, percentiles, and prediction intervals were determined using the same method as in the previous section. We determined on-body measurement uncertainty using calibration measurements in terms of the $50 \%$ and $95 \%$ prediction intervals of the measured incident electric field strength.

\section{Application: Measurement in an Indoor Environment}

The purpose of this indoor measurement campaign was uncertainty assessment of exposure using the two PEMs system applying the on-body calibration for diffuse fields. Moreover, a comparison was made with on-body calibration in the anechoic room and with only the PEM. As mentioned before, indoor environments consist of both diffuse and specular fields so both calibrations are necessary. The same subject, wearing two PEMs, performed a pre-defined walk (for 20 min, 10 times repeating the path between "A" and "B") as shown in Figure 3, in an indoor environment in the second floor of an office building in Ghent (WiFi access points are indicated by a triangle). The WiFi-5G frequency band was considered in this study, because this is an indoor source that emits strong signals in the building. The robust regression on order statistics (ROS) [Helsel, 2005] was applied to process censored data (lower than sensitivity level of PEMs) in which a log-normal distribution was fitted to probability of data above PEM's sensitivity level (Fig. 1). Then average over the two PEMs was calculated. Next, these data were corrected for presence of human body, using calibration factors obtained from the on-body calibration of PEMs in the anechoic room and the RC as:

$$
\frac{E_{R M S, i j}^{b o d y}\left(f_{j}\right)}{\sqrt{p_{50}\left(R_{i j}\left(f_{j}, \varphi\right)\right)}}
$$

\section{RESULTS AND DISCUSSION}

\section{On-Body Responses of Dipoles: Simulation}

Figure 4 shows boxplots of received power on dipole antennas simulated on the front and back of the VFM model for diffuse exposure. Also, average power over two PEMs on the front and back is shown. The box indicates $P I_{50}$ bounded by the $p_{25}$ and $p_{75}$, the bullet indicates the median value $\left(p_{50}\right)$ and whiskers indicate upper and lower adjacent values (values between $p_{75}$ and $p_{75}+1.5 \times P I_{50}$ and all values between $p_{25}$ and $p_{25}-1.5 \times P I_{50}$, respectively). Figure 4 shows that averaging over two antennas reduces variation on the response, for all frequency bands. Table 2 shows statistics of received power averaged over two dipoles (diffuse exposure) on the front and back of the VFM model. For example, Figure 4 shows that for GSM 900-DL the interquartile distance is reduced from $7 \mathrm{~dB}$ for the front and $6.9 \mathrm{~dB}$ for the back to $4.4 \mathrm{~dB}$ for the average over two PEMs (Table 2, column 5). For GSM1800DL the interquartile distance reduces from $6.9 \mathrm{~dB}$ (front) and $7.2 \mathrm{~dB}$ (back) to $4.4 \mathrm{~dB}$ (Fig. 4 and Table 2) for average value. As expected, it can be seen that taking the average value over two antennas for diffuse fields can reduce the uncertainty (variation) of measurement data. 


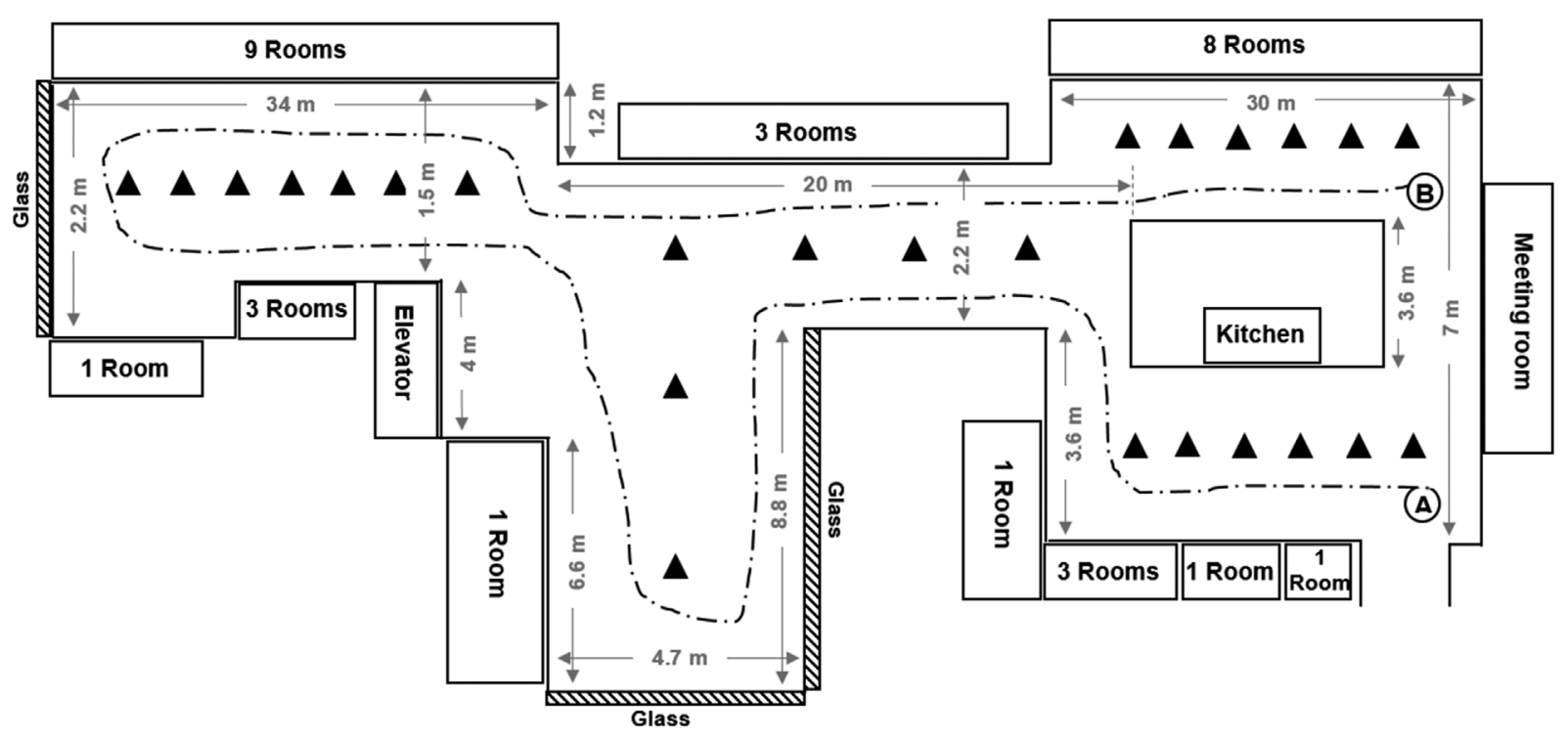

Fig. 3. Ground plan of office building used for indoor measurements. Dashed-dot line indicates route for pre-defined walk. (Triangles show location of access points; the distance between each access point is between $4 \mathrm{~m}$ to $6 \mathrm{~m}$ on average.)

Another estimation of uncertainty (variation) of a PEM's response is the $95 \%$ prediction interval $\left(P I_{95}\right)$. Values of the $P I_{95}$ are $13.5 \mathrm{~dB}$ for UMTS-DL $(2140 \mathrm{MHz})$ and $13.9 \mathrm{~dB}$ for $\mathrm{WiFi}-2 \mathrm{G}(2450 \mathrm{MHz})$, respectively, for an average over two PEMs (Table 2, Column 8). Iskra et al. [2011] reported a $P I_{95}$ of $10.8 \mathrm{~dB}$ at $2100 \mathrm{MHz}$ and Thielens et al. [2015b] reported a $P I_{95}$ of $14.5 \mathrm{~dB}$ at $2450 \mathrm{MHz}$ for a combination of two PEMs on-body. $P I_{95}$ values are also reported for single PEMs: $25.6 \mathrm{~dB}$ at $2100 \mathrm{MHz}$ Iskra et al. [2011] (our study, front: $21.1 \mathrm{~dB}$, back:

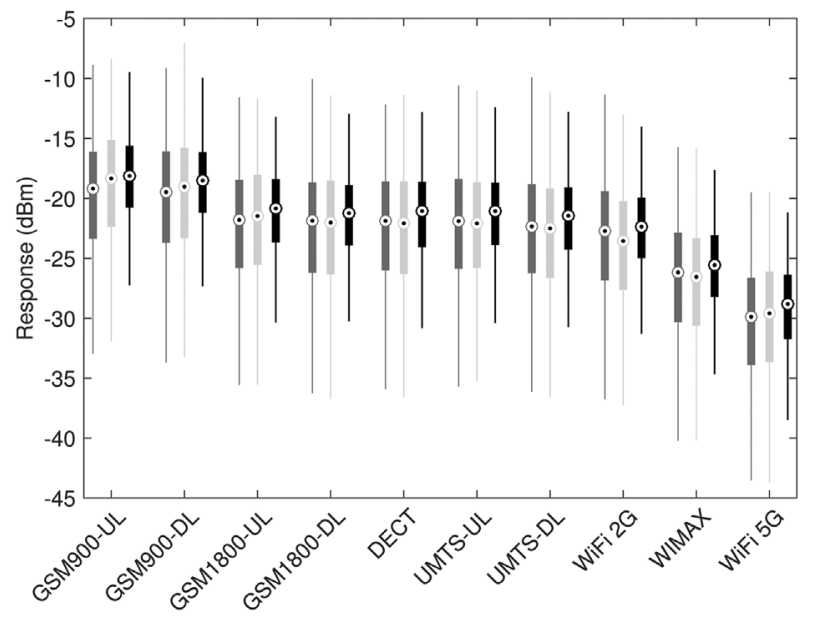

Fig. 4. Boxplots of simulated received powers on dipole antennas on front (dark gray) and back (light gray) of VFM model, and averaged over front and back (black).
$20.6 \mathrm{~dB})$ and $33.8 \mathrm{~dB}$ at $2450 \mathrm{MHz}$ Thielens et al. [2015b] (our study, front: $22.7 \mathrm{~dB}$, back: $21.7 \mathrm{~dB}$ ). The $P_{95}$ values for the average over two PEMs are in good agreement with literature. Individual PEMs show lower values than what is found in literature. The difference might be attributed to the diffuse exposure (vs. specular exposure in Iskra et al. [2011] and Thielens et al. [2015b]) and presence of dipole antennas in the simulation.

\section{Measured On-Body Responses of PEMs: Specular Component in the Anechoic Chamber}

Figure 5 shows boxplots of the distribution of measured responses of PEMs (on front and back of subject's body) in the anechoic chamber for studied frequency bands. Figure 5 confirms experimentally that using two PEMs on the front and back of the body, reduces the uncertainties of their response $\left(P I_{50}\right)$ significantly. For example, for GSM900-UL the interquartile distance is reduced from 11.2 and $14.3 \mathrm{~dB}$ for the front, and back of the body, respectively, to $3 \mathrm{~dB}$ for their average response, which is a considerable improvement (see also Table 2, column 6: AN). Thielens et al. [2015a] reported measurements in the same band and a reduction in $P I_{50}$ from $6.2 \mathrm{~dB}$ and $6.4 \mathrm{~dB}$ (for PEMs on the right and left hip, respectively) to $3.3 \mathrm{~dB}$ for the average response. Results are also comparable with those presented in Bolte et al. [2011]. For other studied frequency bands a significant reduction is observed as well. Each single PEM has a large $\mathrm{PI}_{50}$ value (in the range of 
TABLE 2. Statistics of the Responses Averaged $\left(R_{a v g, j}\right)$ Over Two PEMs for the Studied Frequency Bands

\begin{tabular}{|c|c|c|c|c|c|c|c|c|c|}
\hline \multirow[b]{2}{*}{ RF signal } & \multirow[b]{2}{*}{ Center frequency $(\mathrm{MHz})$} & \multicolumn{2}{|c|}{$p_{50} \quad(R)$} & \multicolumn{3}{|c|}{$\mathrm{PI}_{50}(\mathrm{~dB})$} & \multicolumn{2}{|c|}{$\mathrm{PI}_{95}(\mathrm{~dB})$} & \multirow{2}{*}{$\frac{\text { Ratio of } p_{50}(R)}{\left(\mathrm{R}_{\mathrm{RC}} / \mathrm{R}_{\mathrm{AN}}\right)^{-1}}$} \\
\hline & & AN & $\mathrm{RC}$ & SIM & $\mathrm{AN}$ & $\mathrm{RC}$ & SIM & $\mathrm{RC}$ & \\
\hline GSM900-UL & 897 & 0.15 & 0.07 & 4.5 & 3 & 2.8 & 13.1 & 9.1 & 2.14 \\
\hline GSM900-DL & 950 & 0.17 & 0.06 & 4.4 & 1.9 & 3 & 13.7 & 8.7 & 2.83 \\
\hline GSM1800-UL & 1748 & 0.29 & 0.13 & 4.6 & 2.3 & 2.7 & 13.7 & 7.4 & 2.23 \\
\hline GSM1800-DL & 1843 & 0.27 & 0.09 & 4.4 & 2.4 & 2.6 & 14.2 & 8 & 3 \\
\hline DECT & 1890 & 0.03 & 0.01 & 4.8 & 5.8 & 3 & 14.1 & 7.6 & 3 \\
\hline UMTS-UL & 1950 & 0.32 & 0.08 & 4.5 & 2.5 & 2.8 & 13.1 & 8.2 & 4 \\
\hline UMTS-DL & 2140 & 0.38 & 0.08 & 4.5 & 1.7 & 2.5 & 13.5 & 8.2 & 4.75 \\
\hline WiFi-2G & 2450 & 0.62 & 0.13 & 4.4 & 2.9 & 2.3 & 13.9 & 6.9 & 4.77 \\
\hline WIMAX & 3500 & 0.23 & 0.08 & 4.4 & 4.5 & 2.9 & 13.5 & 7.8 & 2.87 \\
\hline WiFi-5G & 5500 & 0.29 & 0.14 & 4.7 & 4.2 & 2.2 & 13.6 & 6.9 & 2.07 \\
\hline
\end{tabular}

The ratio of the responses is defined as inverse of median response for on-body calibration in RC with respect to on-body calibration in anechoic room. AN, measurement in anechoic room; RC, measurement in reverberation chamber; SIM, simulations of diffuse exposure.

6.9-20.2 dB). These $\mathrm{PI}_{50}$ values are reduced to $2-4 \mathrm{~dB}$ by averaging measurements over two PEMs (Table 2, column 6: AN).

Median of average response (Fig. 5) ranges from $-2 \mathrm{~dB}$ (for WiFi-2G) to $-14.3 \mathrm{~dB}$ (for DECT), which shows that even using a combination of two PEMs underestimates (the median values are $<0 \mathrm{~dB}$ ) the incident electric fields (for specular fields). This is in agreement with Bolte et al. [2011] and Thielens et al. [2015a] and reinforces the necessity of an on-body calibration, also in diffuse fields. Simulation results also presented in Neubauer et al. [2010] and Iskra et al. [2011] also reported an underestimation of average responses of the PEMs by taking the influence of polarization into account. It is shown that averaging over two

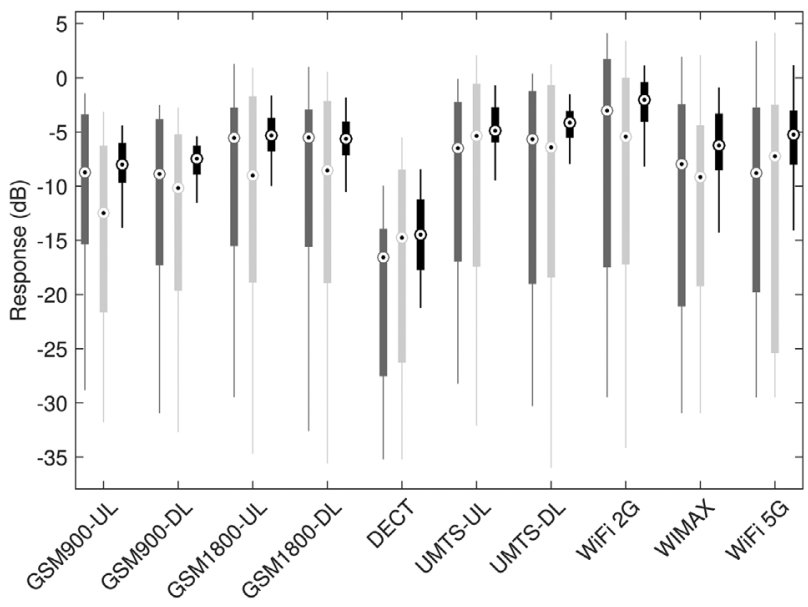

Fig. 5. Boxplots of average response of PEMs for on-body calibration in anechoic chamber (front: dark gray; back: light gray; average: black).
PEMs reduces the uncertainty of response. Since PEMs are on the opposite side of the body, they show a different behavior as a function of the angle of incidence due to shadowing of the body. In other words, the PEM on the front is not correlated with the one on the back. Calculating the correlation coefficients between responses on front and back of VFM confirms the above statement (between front and back: -0.74 at $2450 \mathrm{MHz}$ and -0.49 at $5500 \mathrm{MHz}$, respectively). Also the variance of an average of two responses can be calculated as:

$$
\begin{aligned}
\operatorname{Var}\left(\frac{R_{f r}\left(f_{j}\right)+R_{b k}\left(f_{j}\right)}{2}\right) & =\frac{1}{4}\left(\operatorname{Var}\left(R_{f r}\left(f_{j}\right)\right)+\operatorname{Var}\left(R_{b k}\left(f_{j}\right)\right)\right) \\
& +\frac{1}{2} \operatorname{Cov}\left(R_{f r}\left(f_{j}\right), R_{b k}\left(f_{j}\right)\right)
\end{aligned}
$$

where $\operatorname{Var}(), \operatorname{Cov}(), R_{f r}$, and $R_{b k}$ are variance and covariance of responses, and responses of PEMs on the front and the back of the body, respectively. According to the above equation, averaging the two responses reduces variance of averaged response. Also for two opposite PEMs, their covariance is negative $(-0.33$ at $2450 \mathrm{MHz}$ and -0.1 at $5500 \mathrm{MHz}$ ), which also decreases variance of averaged response. Based on Figure 5, DECT has the lowest response. This is due to the crosstalk as reported in Thielens et al. [2015a]. Crosstalk shows the fraction of power received in a frequency band different from the band that the signal is emitted. For this case, part of the signal emitted in the DECT band is registered in GSM1800-DL and UMTS-UL bands. The former has a crosstalk (non-zero off diagonal element of crosstalk matrix) of 0.18 while the latter has a crosstalk of 0.035 . 


\section{Measured On-Body Responses of PEMs: Diffuse Component in RC}

Figure 6 shows boxplots of responses of the PEMs on the front and back of the subject's body obtained from calibration in RC. Also Table 2 lists $P I_{50}$ of average response over two PEMs for on-body calibrations in RC. Small variations around $4 \mathrm{~dB}$ are obtained for $P I_{50}$ of each PEM on the front or back of the body. Compared to the calibration in the anechoic room, uncertainty for each PEM and average response is reduced. Figure 6 shows, for example, $P I_{50}$ for GSM900-UL is $4.2 \mathrm{~dB}$ (PEM front), $4.9 \mathrm{~dB}$ (PEM back) and $2.8 \mathrm{~dB}$ (averaged over two PEMs) compared to $11.2 \mathrm{~dB}$ (PEM front) and $14.3 \mathrm{~dB}$ (PEM back) and an average response of $3 \mathrm{~dB}$ in the anechoic room (Table 2, columns 6 and 7). Another example is UMTS-DL for which $P I_{50}$ improved from $3.9 \mathrm{~dB}$ (PEMs on front and back, see Fig. 6) to $2.5 \mathrm{~dB}$ (average) compared to $16.8 \mathrm{~dB}$ (PEMs on the front and back) and an average response of $1.7 \mathrm{~dB}$ in the anechoic room. As mentioned before, averaging responses over two PEMs in diffuse fields reduces variation in their median response. Similar to anechoic chamber and simulations, median values of the average response for all frequency bands are lower than $0 \mathrm{~dB}$, showing also an underestimation for diffuse fields when measurements with PEMs are performed [Bolte et al., 2011; Thielens et al., 2015a]. The minimum value of underestimation is for DECT $(-18.24 \mathrm{~dB})$ due to the cross talk with GSM1800-DL (off-diagonal element: 0.065) and UMTS-UL (offdiagonal element: 0.012) bands. Except DECT band, median of the average response is in the range of -8.5 to $-12.2 \mathrm{~dB}$.

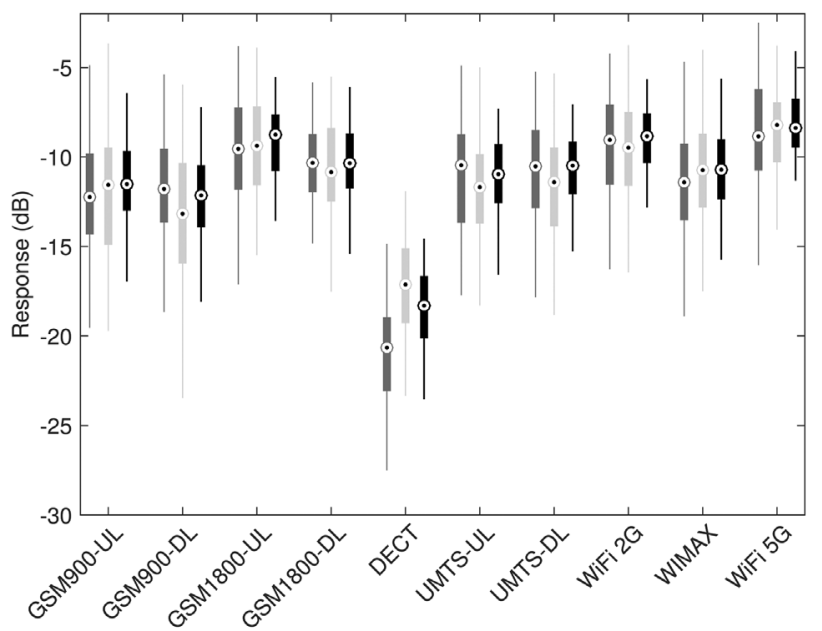

Fig. 6. Boxplots of average response of PEMs for on-body calibration in reverberation chamber (front: dark gray; back: light gray; average: black).
The ratio of on-body calibration in anechoic room and $\mathrm{RC}$ is defined and listed in Table 2 as inverse of median response (averaged over two PEMs) for on-body calibration in RC with respect to response obtained from on-body calibration in the anechoic room $\left(R_{R C} / R_{A N}\right)^{-1}$. This ratio shows that using the on-body calibration in anechoic room, the two-PEM measurement system, underestimates the actual diffuse exposure in indoor environments by a factor of 2 to 4 for each studied frequency band. This can be compensated by using the on-body response obtained from on-body calibrations in the RC. Moreover, it must be noted that validation measurements cannot be done for real signals separately since it is impossible to record field values on-body and in the absence of the person at the same time and on the same location. Therefore, the above ratio is used as a parameter for comparison, since it considers the incident field values both in the presence and in the absence of the person.

Also from Table 2, it can be seen that $P I_{50}$ values for simulations are close but higher than the $P I_{50}$ of measurement data in $\mathrm{RC}$, with a mean absolute deviation of $1.83 \mathrm{~dB}$ which is a very good agreement. Also the mean absolute deviation between $\mathrm{PI}_{95}$ (of the average response) of measurements in $\mathrm{RC}$ and simulation data is $5.76 \mathrm{~dB}$. It must be noted that this is a logical comparison between $P I_{50}$ and $P I_{95}$ values, since we did not model the exact RC in simulations (dimensions of the RC and real PEMs including their antenna were not considered in simulations). This shows that numerical simulations are a good model for diffuse fields.

Using the NBM-550 field meter in combination with EF 0391/EF 0691 probes causes a standard uncertainty of $1.1 \mathrm{~dB}$ on the free-space measurements during the calibration measurements in both the anechoic and reverberation chambers (see step 1: onbody calibration measurements). The reverberation chamber has a total field standard deviation of $\leq 1.5 \mathrm{~dB}$ (below the $3 \mathrm{~dB}$ tolerance limit) in the range of $800-1000 \mathrm{MHz}$ that decreases by increasing frequency.

\section{Application: Measurement in an Indoor Environment}

Figure 7 shows measured incident electric field averaged over two PEMs determined by on-body calibration in anechoic room, and by on-body calibration in the RC and is compared with measurement data of PEMs (no body calibration/only PEMs). Measured data (67\% and 53\%) were censored (below detection limit of the PEMs: $0.02 \mathrm{~V} / \mathrm{m}$ at $5500 \mathrm{MHz}$ ) for the PEM on the front and the back, respectively. 


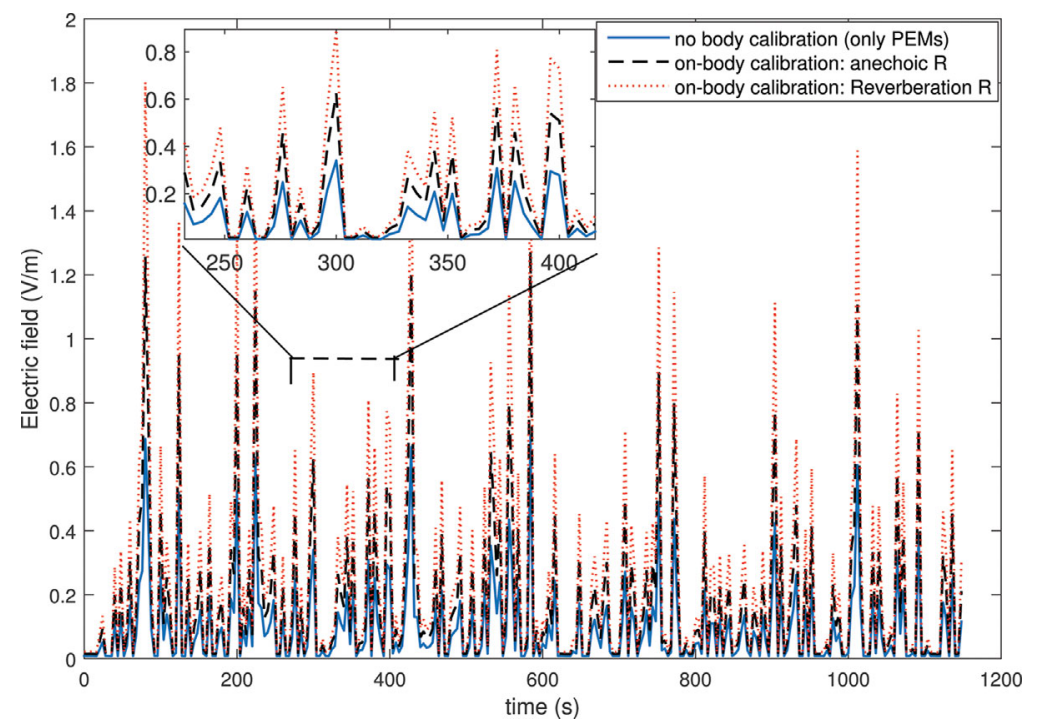

Fig. 7. Incident electric field strength results, measured in office averaged over two PEMs and applying free-space (no body calibration/only PEMs), anechoic room and RC calibrations. Anechoic $\mathrm{R}$ and reverberation $\mathrm{R}$ mean anechoic chamber and reverberation room, respectively.

Peaks are due to the presence of wireless routers and access points in the building (see indication on ground plan in Fig. 3). The result for on-body calibration in the $\mathrm{RC}$ is higher than results of no-body calibration (only PEMs/calibration in absence of a person) and on-body calibration in anechoic room. The actual incident field value should be in between both estimates but its exact value cannot be calculated, since the contribution of specular and diffuse fields in real environments is not known. The median electric field of $0.04 \mathrm{~V} / \mathrm{m}, 0.074 \mathrm{~V} / \mathrm{m}$, and $0.11 \mathrm{~V} / \mathrm{m}$ is measured for only PEMs, anechoic room and RC calibrations, respectively. These measured values satisfy issued reference levels $(61 \mathrm{~V} / \mathrm{m}$ for the considered WiFi frequency of $5 \mathrm{GHz}$ ) by ICNIRP [1998] for the general public. Summary statistics of the measured incident power densities $\left(S_{\text {inc }}\right)$ are presented using Table 3. The ratio of incident power density is proposed for the median incident power density of the three calibrations. The no body calibration (only PEMs) clearly underestimates the on-body measurements by a factor of $7.55\left(S_{i n c, R C} / S_{\text {inc, free }}\right)$. The on-body anechoic room calibration will also underestimate actual exposure to diffuse fields as RC results in higher values (factor of $2.21\left[S_{i n c, R C} / S_{i n c, A N}\right]$ ). Maximum measured incident power density is for RC calibration (at $t=584 \mathrm{~s}) 8.9 \mathrm{~mW} / \mathrm{m}^{2}$, which is lower than the ICNIRP reference levels $\left(10 \mathrm{~W} / \mathrm{m}^{2}\right.$ at $\left.5 \mathrm{GHz}\right)$ for the general public. We advise RC calibration for indoor measurements using PEMs on body, as no body calibration (only PEMs) and anechoic room calibration underestimate actual exposure.

TABLE 3. Summary Statistics of Measured Incident Power Density in the Office at the WiFi-5G Band

\begin{tabular}{|c|c|c|c|c|c|}
\hline RF Signal & Front & Back & Average (PEMs) & Average (AN) & Average (RC) \\
\hline Censored data $(\%)$ & 67.01 & 52.77 & - & - & - \\
\hline$\left(\mathrm{S}_{\text {inc, min }}\right)\left(\mu \mathrm{W} / \mathrm{m}^{2}\right)$ & 0.13 & 0.13 & 0.13 & 0.44 & 1.06 \\
\hline$\left(\mathrm{S}_{\text {inc, max }}\right)\left(\mathrm{mW} / \mathrm{m}^{2}\right)$ & 4.1 & 3.9 & 1.3 & 4.3 & 8.9 \\
\hline$\left(\mathrm{S}_{\text {inc, average }}\right)\left(\mu \mathrm{W} / \mathrm{m}^{2}\right)$ & 13 & 38.84 & 24.45 & 81.23 & 165.8 \\
\hline $\mathrm{p}_{25}\left(\mathrm{~S}_{\mathrm{inc}}\right)\left(\mu \mathrm{W} / \mathrm{m}^{2}\right)$ & 0.13 & 0.17 & 0.17 & 0.6 & 1.17 \\
\hline $\mathrm{p}_{50}\left(\mathrm{~S}_{\mathrm{inc}}\right)\left(\mu \mathrm{W} / \mathrm{m}^{2}\right)$ & 0.17 & 0.17 & 4.25 & 14.52 & 32.1 \\
\hline $\mathrm{P}_{75}\left(\mathrm{~S}_{\mathrm{inc}}\right)\left(\mu \mathrm{W} / \mathrm{m}^{2}\right)$ & 9.23 & 87.86 & 52 & 179.3 & 363.1 \\
\hline$p_{50}\left(\mathrm{~S}_{\mathrm{inc}, \mathrm{RC}} / \mathrm{S}_{\mathrm{inc}, \mathrm{AN}}\right)$ & & & 2.21 & & \\
\hline$p_{50}\left(\mathrm{~S}_{\text {inc, RC }} / \mathrm{S}_{\text {inc, FR-SP }}\right)$ & & & 7.55 & & \\
\hline
\end{tabular}

PEMs, no-body calibration (only PEMs); AN, on-body calibration in anechoic room; RC, on-body calibration in reverberation room. 


\section{CONCLUSIONS}

In this article, for the first time, the response of two PEMs in diffuse fields is studied both numerically and experimentally. Two PEMs worn on the body were calibrated in an anechoic room and in a reverberation chamber, and actual fields were compared with and without the human body, under non-diffuse and diffuse field exposures, respectively. Numerical simulations were used to study response of PEMs. However an exact comparison is not possible; the interquartile distance of responses obtained from simulations is close to the values obtained for on-body calibrations. All results, whether using numerical simulations or calibration measurements in both setups, show that using a combination of two PEMs on-body reduces uncertainty of measurements up to $3 \mathrm{~dB}$ compared to single PEMs. DECT has the lowest response among other frequency bands, which is due to the crosstalk with GSM1800-DL and UMTS-UL bands. As an application, indoor WiFi5G was measured in an office building in Ghent, Belgium. To the best of the authors' knowledge, this frequency band has not often been investigated yet and to date, no on-body calibration measurements using PEMs has been performed in this band. Three calibrations, no body calibration (only PEMs), anechoic room, and reverberation room, are applied to measured data. All measured power density values (maximally $8.9 \mathrm{~mW} /$ $\mathrm{m}^{2}$ and on average $165.8 \mu \mathrm{W} / \mathrm{m}^{2}$ for on-body calibration in RC) satisfy issued reference levels by ICNIRP. Power density values obtained from on-body calibration in RC are higher than values obtained from only PEMs and on-body calibration in anechoic room, by factors of 7.55 and 2.21, respectively. Also it is shown that the median response averaged over two PEMs for RC calibrations is higher than the response for anechoic room calibrations by a factor of 2 to 4 for studied frequency bands. This indicates that previously reported PEM measurements in anechoic room underestimate actual exposure to diffuse fields in indoor environments $(0.04 \mathrm{~V} / \mathrm{m}$, $0.074 \mathrm{~V} / \mathrm{m}$ and $0.11 \mathrm{~V} / \mathrm{m}$ for only PEMs, on-body calibration in anechoic chamber and reverberation chamber, respectively). We advise RC calibration for indoor measurements using PEMs on body. Future research will consist of using on-body antennas (personal distributed exposimeter calibrated on-body in RC) instead of commercial PEMs.

\section{REFERENCES}

Andersen JB, Nielsen JØ, Pedersen GF, Bauch G, Herdin M. 2007. Room electromagnetics. IEEE Antennas Propag Mag 49:27-33.

Andersen JB, Chee KL, Jacob M, Pedersen GF, Kurner T. 2012. Reverberation and absorption in an aircraft cabin with the impact of passengers. IEEE Trans Antennas Propag 60:2472-2480.

Bahillo A, Blas J, Fernandez P, Lorenzo RM, Mazuelas S, Abril EJ. 2008. E-field assessment errors associated with RF dosimeters for different angles of arrival. Radiat Prot Dosim 123:51-56.

Balanis CA. 2005. Antenna theory: Analysis and design. 3rd ed. Hoboken, NJ: Wiley. pp 93-94.

Bamba A, Joseph W, Andersen JB, Tanghe E, Vermeeren G, Plets D, Nielsen G, Martens L. 2012. Experimental assessment of specific absorption rate using room electromagnetics. IEEE Trans Electromagn Compat 54:747-757.

Bamba A, Joseph W, Vermeeren G, Tanghe E, Gaillot DP, Andersen JB, Nielsen G, Lienard M, Martens L. 2013. Validation of experimental whole-body SAR assessment method in a complex environment. Bioelectromagnetics 34:122-132.

Bamba A, Gaillot DP, Tanghe E, Vermeeren G, Joseph W, Lienard M, Martens L. 2015. Assessing whole-body absorption cross section for diffuse exposure from reverberation chamber measurements. IEEE Trans Electromagn Compat 57:27-34.

Belgian Institute for Postal Services and Telecommunications (BIPT). 2015. Available at http://www.bipt.be [Last accessed 1 April 2015].

Bolte JFB, Van der Zande G, Kamer J. 2011. Calibration and uncertainties in personal exposure measurements of radiofrequency electromagnetic fields. Bioelectromagnetics 32:652-663.

Bolte JFB, Eikelboom T. 2012. Personal radiofrequency electromagnetic field measurements in the Netherlands: Exposure level and variability for everyday activities, times of day and types of area. Environ Int 48:133-142.

Christ A, Kainz W, Hahn EG, Honegger K, Zefferer M, Neufeld E, Rascher W, Janka R, Bautz W, Chen J, Kiefer B, Schmitt P, Hollenbach H, Shen J, Oberle M, Szczerba D, Kam A, Guag JW, Kuster N. 2010. The virtual family-development of surface-based anatomical models of two adults and two children for dosimetric simulations. Phys Med Biol 55: N23-N38.

Gabriel C, Gabriel S, Corthout E. 1996. The dielectric properties of biological tissues. Phys Med Biol 41:2231-2293.

Helsel DR. 2005. In: Scott M, Barnett V, editors. Nondetects and data analysis. Hoboken, New Jersey: John Wiley \& Sons. Chapter 6.

International Commission on Non-Ionizing Radiation Protection (ICNIRP). 1998. Guidelines for limiting exposure to timevarying electric, magnetic, and electromagnetic fields (up to $300 \mathrm{GHz}$ ). Health Phys 74:494-522.

Iskra S, McKenzie R, Cosic I. 2010. Factors influencing uncertainty in measurement of electric fields close to the body in personal RF dosimetry. Radiat Prot Dosim 140:25-33.

Iskra S, McKenzie R, Cosic I. 2011. Monte Carlo simulations of the electric field close to the body in realistic environments for application in personal radiofrequency dosimetry. Radiat Prot Dosim 147:517-527.

Joseph W, Vermeeren G, Verloock L, Heredia MM, Martens L. 2008. Characterization of personal RF electromagnetic field exposure and actual absorption for the general public. Health Phys 95:317-330.

Joseph W, Frei P, Röösli M, Thuroczy G, Gajsek P, Trcek T, Bolte J, Vermeeren G, Mohler E, Juhasz P, Finta V, Martens L. 2010. Comparison of personal radio frequency 
electromagnetic field exposure in different urban areas across Europe. Environ Res 110:658-663.

Mann S. 2010. Assessing personal exposures to environmental radiofrequency electromagnetic fields. CR Phys 11:541-555.

Neubauer G, Cecil S, Giczi W, Petric B, Preiner P, Fröhlich J, Röösli M. 2010. The association between exposure determined by radiofrequency personal exposimeters and human exposure: A simulation study. Bioelectromagnetics 31:535-545.

Poutanen J, Salmi J, Haneda K, Kolmonen VM, Vainikainen P. 2011. Angular and shadowing characteristics of dense multipath components in indoor radio channels. IEEE Trans Antennas Propag 59:245-253.

Roelens L, Joseph W, Reusens E, Vermeeren G, Martens L. 2008. Characterization of scattering parameters near a flat phantom for wireless body area networks. IEEE Trans Electromagn Compat 50:185-193.

Röösli M, Frei P, Mohler E, Braun-Fahrländer C, Bürgi A, Fröhlich J, Neubauer G, Theis G, Egger M. 2008. Statistical analysis of personal radiofrequency electromagnetic field measurements with non-detects. Bioelectromagnetics 29:471-478.
Röösli M, Frei P, Bolte J, Neubauer G, Cardis E, Feychting M, Gasjek P, Heinrich S, Joseph W, Mann S, Martens L, Mohler E, Parslow RC, Poulsen AH, Radon K, Schüz J, Thuroczy G, Viel J, Vrijheid M. 2010. Conduct of a personal radiofrequency electromagnetic field measurement study: Proposed study protocol. Environ Health 9:23.

SPEAG. 2009. SEMCAD X Reference Manual. Schmid \& Partner Enginnering AG, Zurich, Switzerland. Available at http:// www.semcad.com [Last accessed 1 April 2015].

Thielens A, De Clercq H, Agneessens S, Lecoutere J, Verloock L, Declercq F, Vermeeren G, Tanghe E, Rogier H, Puers R, Martens L, Joseph W. 2013. Personal distributed exposimeters for radio frequency exposure assessment in real environments. Bioelectromagnetics 34:563-567.

Thielens A, Agneessens S, Verloock L, Tanghe E, Rogier H, Martens L, Joseph W. 2015a. On-body calibration and processing for a combination of two radio-frequency personal exposimeters. Radiat Prot Dosim 163:58-69.

Thielens A, Agneessens S, De Clercq H, Lecoutere J, Verloock L, Tanghe E, Aerts S, Puers R, Rogier H, Martens L, Joseph W. 2015b. On-body calibration and measurements using a personal, distributed exposimeter for wireless fidelity. Health Phys 108:407-418. 